\title{
A New Approach to Non-termination Analysis of Logic Programs
}

\author{
Dean Voets` and Danny De Schreye \\ Department of Computer Science, K.U.Leuven, Belgium \\ Celestijnenlaan 200A, 3001 Heverlee \\ $\{$ Dean.Voets, Danny. DeSchreye\}@cs.kuleuven. be
}

\begin{abstract}
In this paper, we present a new approach to non-termination analysis of logic programs, based on moded SLDNF-resolution. Moded SLDNF-resolution is a symbolic execution for moded goals, developed for termination prediction. To prove non-termination, we use a complete loop checker to create a finite symbolic derivation tree of a logic program for a moded query. Then, we check if this derivation tree contains an infinite loop, using a new non-termination condition. We implemented this approach and tested it on the benchmark from the Termination Competition of 2007. The results are very satisfactory: our tool is able to prove non-termination and construct non-terminating queries for all non-terminating benchmark programs, and thus, significantly improves on the results of the only other non-termination analyzer, NTI.
\end{abstract}

Keywords: non-termination analysis, program analysis.

\section{Introduction}

One of the central concerns of declarative programming, in particular of Logic Programming, is that the use of a declarative programming style in a declarative programming language leads to less error-prone, more understandable and better maintainable programs. However, it is well-known that a declarative programming style also results in less efficient computations, and in the extreme case, in non-terminating computations. The latter problem has received considerable attention within the community. Much research has been done on termination analysis, loop detection and more recently, non-termination analysis.

Among these areas, termination analysis has by far received most attention. Most of the more powerful approaches and techniques have been introduced in the last decade: the constraint-based approach to termination analysis [6], the local approaches [4, the use of types in termination analysis [2], powerful transformational approaches [11, termination inference [8], and the porting of TRS-techniques to the LP-context [9].

A rather recent concern in this research is the precision of the termination analysis. Since termination is undecidable in general, only sufficient conditions for termination are verified. It is important to have a good understanding of the precision of these techniques: do they actually capture most of the terminating computations?

\footnotetext{
* Supported by the Fund for Scientific Research - FWO-project G0561-08.
} 
With respect to the other two approaches, loop detection and non-termination analysis, there is often confusion concerning their relation. Because both approaches use similar techniques, their distinguishing features and aims are not always well understood. Loop detection is a run-time technique. It aims to cut infinite derivations for a concrete query at run-time. For an extensive overview and comparison of different loop checking algorithms, we refer to [1. Non-termination analysis is a compile-time approach. It aims to prove that a certain class of queries will result in non-terminating computations for at least some of the queries in the considered class. Non-termination analysis is performed for classes of queries described in terms of modes (or types). One of the key concerns of non-termination analysis is to address the important issue of precision analysis of termination analysis. A termination analysis can be shown to be precise by proving that the class of queries for which termination could not be proven is actually non-terminating. This has been one of the main goals and achievements of the only non-termination analyzer developed up till now, NTI 10.

Very recently, yet another, fourth approach to the problem has been introduced: termination and non-termination prediction [12. In this approach, techniques developed in loop-detection are lifted to classes of moded queries to allow for a prediction of the termination behavior of these queries. Although the predictions do not take the form of formal proofs, experiments show that they can be extremely precise. Moreover, for non-termination prediction, it has been proven that by increasing a parameter in the analysis, the repetition number, in the limit, the prediction is always correct.

Our work has been inspired both by the work on termination/non-termination prediction and by NTI. We propose a new non-termination analysis. It reuses the analysis scheme proposed in [12] to produce a finite representation of the computation for a moded query, given some logic program. We introduce a new nontermination condition expressed in terms of this finite representation of the computation. We prove its correctness and extend it to increase its applicability.

It turns out that our characterization of non-terminating computations is more precise than that of NTI. We have implemented the technique and performed extensive experiments with it on the basis of the benchmark of the termination analysis competition of 20071. The experiments show that our technique has a $100 \%$ success-rate on this benchmark, outperforming the only competing approach, NTI.

The paper is organized as follows. In the next section we introduce some preliminaries. In section 3, we present our conditions implying non-termination and show that we are able to derive classes of non-terminating queries. In Section 4 , we present our experimental evaluation and we compare our analyzer with the non-termination inference tool NTI [10]. Finally, Section 5 concludes this paper.

\section{Preliminaries}

In this section, we introduce some preliminaries concerning the symbolic derivation trees used to prove non-termination. First, we introduce moded SLDNF-trees

\footnotetext{
${ }^{1}$ http://www.lri.fr/ ${ }^{2}$ marche/termination-competition/
} 
as defined in [12. These trees represent the derivation trees of all concrete queries corresponding to a moded query. Then, we introduce complete loop checks for these SLDNF-trees and introduce LP-check [12, a loop check for moded SLDNFresolution introduced for termination prediction.

\subsection{Moded Generalized SLDNF-Trees}

We assume the reader is familiar with standard terminology of logic programs, in particular with SLDNF-resolution and substitutions, as described in 7]. Variables are denoted by character strings beginning with a capital letter. Predicates, functions and constant symbols are denoted by character strings beginning with a lower case letter. A term is a constant, a variable, or a function of the form $f\left(t_{1}, \ldots, t_{m}\right)$ where $f$ is a function symbol and each $t_{i}$ is a term. We denote the set of terms constructible from a program $P$, by Term $_{P}$. An atom is of the form $p\left(t_{1}, \ldots, t_{m}\right)$ where $p$ is a predicate symbol. Two atoms are called variants if they are equal up to variable renaming. An atom $A$ is more general than an atom $B$, if there exists a substitution $\theta$, such that $A \theta=B$. A literal is an atom $A$ or the negation $\neg A$ of $A$.

A general logic program $P$ is a finite set of clauses of the form $A \leftarrow L_{1}, \ldots, L_{n}$, where $A$ is an atom and each $L_{i}$ is a literal. A goal $G_{i}$ is a headless clause $\leftarrow L_{1}, \ldots, L_{n}$. A query, $Q$, is a conjunction of literals $L_{1}, \ldots, L_{n}$. Without loss of generality, we assume that $Q$ consists only of one atom.

Let $P$ be a logic program and $G_{0}$ a query. $G_{0}$ is evaluated by building a generalized $S L D N F$-tree $G T_{G_{0}}$ as defined in [12, in which each node is represented by $N_{i}: G_{i}$ where $N_{i}$ is the name of the node and $G_{i}$ is a goal attached to the node. We do not reproduce the definition of a generalized SLDNF-tree. Roughly speaking, $G T_{G_{0}}$ is the set of standard SLDNF-trees for $P \cup\left\{G_{0}\right\}$ augmented with an ancestor-descendant relation on their literals. Let $L_{i}$ and $L_{j}$ be the selected literals at two nodes $N_{i}$ and $N_{j}$, respectively. $L_{i}$ is an ancestor of $L_{j}$, denoted $L_{i} \prec_{\text {anc }} L_{j}$, if the proof of $L_{i}$ goes via the proof of $L_{j}$. Throughout the paper, we choose to use the best-known depth-first, left-most control strategy, as is used in Prolog, to select goals and literals. So by the selected literal in each node $N_{i}: \leftarrow L_{1}, \ldots, L_{n}$, we refer to the left-most literal $L_{1}$. For any node $N_{i}: G_{i}$, we use $L_{i}^{1}$ to refer to the selected literal in $G_{i}$.

Recall that in SLDNF-resolution, let $L_{i}=\neg A$ be a ground negative literal selected at $N_{i}$, then, by the negation-as-failure rule [7], a subsidiary child SLDNFtree will be built to solve $A$. In a generalized SLDNF-tree $G T_{G_{0}}$, such parent and child SLDNF-trees are connected from $N_{i}$ to $N_{i+1}$ via a dotted edge "..." ,called a negation arc, and $A$ at $N_{i+1}$ inherits all ancestors of $L_{i}$ at $N_{i}$. Therefore, a path of a generalized SLDNF-tree may come across several SLDNF-trees through dotted edges. Any such path starting at the root node $N_{0}: G_{0}$ of $G T_{G_{0}}$ is called a generalized SLDNF-derivation.

We do not consider floundering queries; i.e., we assume that no non-ground negative literals are selected at any node of a generalized SLDNF-tree (see [12]).

A derivation step is denoted by $N_{i}: G_{i} \Rightarrow_{C} N_{i+1}: G_{i+1}$, meaning that applying a clause $C$ to $G_{i}$ produces $N_{i+1}: G_{i+1}$. 
As stated in the introduction, we want to prove non-termination for classes of queries described using modes. An input mode stands for an arbitrary ground term, i.e. it can be any variable-free term of Term $_{P}$. An output mode stands for a free variable. A query $Q$ is a moded query if some arguments of $Q$ are input modes, otherwise, it is a concrete query. Because an input mode denotes an arbitrary ground term, we may approximate the effect of an input mode, by treating it as a special variable $I$, in such a way that in SLDNF-derivations $I$ can be substituted by a constant or function, but cannot be substituted by an ordinary variable. Therefore, when unifying a special variable $I$ and a variable $X$, we always substitute $I$ for $X$. In the remainder of the paper, we denote a special variable by underlining the variable's name.

Definition 1. Let $P$ be a logic program and $Q=p\left(I_{1}, \ldots, I_{m}, T_{1}, \ldots, T_{n}\right)$ a moded query. The moded generalized SLDNF-tree of $\bar{P}$ for $\bar{Q}$, is defined to be the generalized SLDNF-tree $G T_{G_{0}}$ for $P \cup\left\{\leftarrow p\left(\underline{I_{1}}, \ldots, \underline{I_{m}}, T_{1}, \ldots, T_{n}\right)\right\}$, with each $I_{i}$ being a distinct special variable not occurring in any $T_{j}$. The special variables $\underline{I_{1}}, \ldots, \underline{I_{m}}$ are called input variables.

In a moded generalized SLDNF-tree, an input variable $\underline{I}$ may be substituted by either a constant or a function $f\left(t_{1}, \ldots, t_{n}\right)$. If $\underline{I}$ is substituted by $f\left(t_{1}, \ldots, t_{n}\right)$, all variables in $t_{1}, \ldots, t_{n}$ are also called input variables and treated as special variables. We refer to Figure 1(a) for an illustration of (part of) a moded generalized SLDNF-tree. The figure also illustrates a loop check.

A moded atom $A$ corresponds to a set of concrete atoms, called the denotation of $A$. Let $I_{1}, \ldots, I_{n}$ be all input variables occurring in $A$. Let $t_{1}, \ldots, t_{n} \in$ Term $_{P}$. $A\left(t_{1} \rightarrow \underline{I_{1}}, \ldots, t_{n} \rightarrow I_{n}\right)$ denotes the concrete atom obtained by replacing the input variables $\underline{I_{1}}, \ldots, \overline{I_{n}}$ by the terms $t_{1}, \ldots, t_{n}$.

Definition 2. Let $A$ be an atom with $\underline{I_{1}}, \ldots, \underline{I_{n}}$ as its input variables. The denotation of $A$ is

$$
\operatorname{Den}(A)=\left\{A\left(t_{1} \rightarrow \underline{I_{1}}, \ldots, t_{n} \rightarrow \underline{I_{n}}\right) \mid t_{i} \in \text { Term }_{P}, t_{i} \text { is ground }\right\} .
$$

This concept can be adapted to moded goals in a straightforward way. Note that the denotation of a concrete atom is a singleton containing the atom itself.

\subsection{Loop Checking}

A complete loop check for moded goals cuts all infinite branches in a moded generalized SLDNF-tree.

Definition 3. A loop check $L$ is complete w.r.t. moded SLDNF-resolution if for every logic program $P$ and moded query $Q$, every infinite derivation of $P$ for $Q$ is cut by $L$.

Many simple complete loop checks can be constructed, for example a bound on the number of times a certain predicate occurs in a derivation. However, only one loop check for moded SLDNF-resolution is discussed in the literature, LP-check [12. LP-check is a complete loop check developed for termination prediction. 
In [12, it is proven that every infinite derivation contains an infinite chain of loop goals. These are goals satisfying some conditions on the selected literals. A clause is cut by LP-check if a prefix of such a chain is encountered.

Definition 4. Let $A$ be a moded atom, the symbol string of $A, S_{A}$, is the string obtained by reading all predicate symbols, function symbols, constants and variables in $A$, from left to right, with the variables replaced by $\mathcal{X}$.

A symbol string $S_{A_{1}}$ is a projection of $S_{A_{2}}$, denoted $S_{A_{1}} \sqsubseteq_{p r o j} S_{A_{2}}$, if $S_{A_{1}}$ is obtained from $S_{A_{2}}$ by removing zero or more elements.

Example 1. Let $A_{1}=a$ and $A_{2}=f(X, g(X, f(a, \underline{I})))$. Then, $S_{A_{1}}=a, S_{A_{2}}=$ $f \mathcal{X}$ X faX and $S_{A_{1}} \sqsubseteq_{\text {proj }} S_{A_{2}}$.

Definition 5. Let $N_{i}: G_{i}$ and $N_{j}: G_{j}$ be two nodes in a derivation with $L_{i}^{1} \prec$ anc $L_{j}^{1}$ and $S_{L_{i}^{1}} \sqsubseteq \operatorname{proj} S_{L_{j}^{1}}$. Then, $G_{j}$ is called a loop goal of $G_{i}$.

LP-check uses a repetition number defining how long the chain of loop goals can become before it is cut by LP-check.

Definition 6. Given a repetition number $r \geq 2, \boldsymbol{L P}$-check is defined as follows: Any derivation $D$ in a generalized SLDNF-tree is cut at a node $N_{g_{r}}$ if $D$ has a prefix of the form

$$
N_{0}: G_{0} \Rightarrow_{C_{0}} \ldots N_{g_{1}}: G_{g_{1}} \Rightarrow_{C_{k}} \ldots N_{g_{2}}: G_{g_{2}} \Rightarrow_{C_{k}} \ldots N_{g_{r}}: G_{g_{r}} \Rightarrow_{C_{k}}
$$

such that (a) for any $j<r, G_{g_{j+1}}$ is a loop goal of $G_{g_{j}}$, and (b) for all $j \leq r$, the clause $C_{k}$ applied to $G_{g_{j}}$ is the same. The prefix is called an $\boldsymbol{L P}$-cut, the nodes $N_{g_{1}}, \ldots, N_{g_{r}}$ are called the nodes of the LP-cut.

Because LP-check is a rather expensive loop check, a variant on LP-check is defined in [12: LP-check with pruning. This loop check reduces the amount of redundant branches by pruning clauses if they are already applied to an ancestor or descendant with a variant as a selected literal. We illustrate these loop checks with the binary tree program.

Example 2. The following program succeeds if the argument of the query represents a binary tree.

bin (empty).

$$
\operatorname{bin}\left(\operatorname{tree}\left(L,{ }_{-}, R\right)\right):-\operatorname{bin}(L), \operatorname{bin}(R) .
$$

Figures 1(a) and 1(b) show moded SLDNF-trees constructed using LP-check and LP-check with pruning, respectively, for the binary tree program with $\operatorname{bin}(\underline{I})$ as a query and 3 as a repetition number.

In the SLDNF-tree constructed by LP-check, as shown in Figure 1(a), clause 2 is cut at node $N_{5}$ because of LP-cut: $N_{0} \Rightarrow_{C_{2}} \ldots N_{3} \Rightarrow_{C_{2}} N_{5} \Rightarrow_{C_{2}}$. Similarly, LP-check cuts clause 2 at nodes: $N_{6}, N_{8}, N_{9}, N_{12}$ and $N_{13}$.

The SLDNF-tree constructed by LP-check with pruning, depicted in Figure 1(b), is much smaller. Clause 1 is cut at nodes $N_{2}$ and $N_{3}$, because that clause is applied to the selected literal of $N_{0}$, which is both an ancestor and a variant of the selected literals of nodes $N_{2}$ and $N_{3}$. At node $N_{3}$, clause 2 is cut by LP-check with pruning because of LP-cut: $N_{0} \Rightarrow_{C_{2}} N_{2} \Rightarrow_{C_{2}} N_{3} \Rightarrow_{C_{2}}$. 


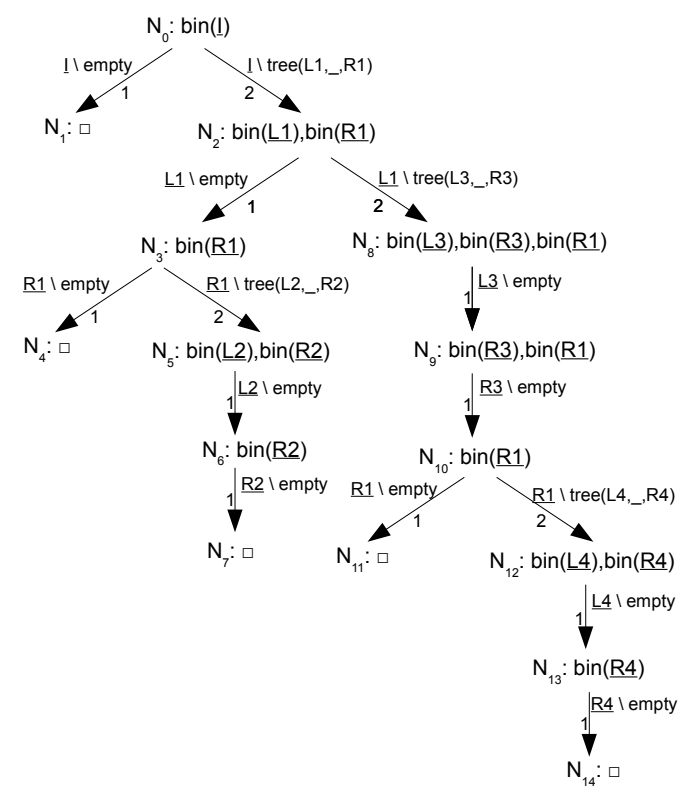

(a) LP-check

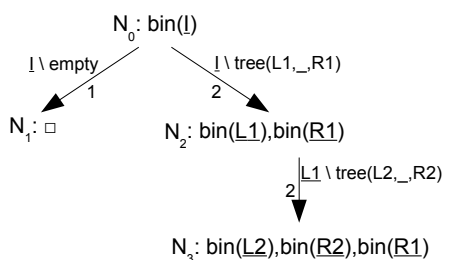

$\mathrm{N}_{3}: \operatorname{bin}(\underline{\mathrm{L}} 2), \operatorname{bin}(\underline{\mathrm{R} 2}), \operatorname{bin}(\underline{\mathrm{R} 1})$

Fig. 1. Two loop checks for moded SLDNF-resolution

\section{A New Non-termination Condition}

In this section, we present a new non-termination analysis technique for general logic programs with moded queries. We consider a program non-terminating w.r.t. a moded query, if the denotation of the query contains at least one concrete query that has an infinite branch in its generalized SLDNF-tree.

\subsection{The Moded More General Relation}

To prove non-termination, we prove that a path between two nodes $N_{b}$ and $N_{e}$ in a moded SLDNF-derivation can be repeated infinitely often. To find such a path, we check three properties. Because the rules in the path must be applicable independent of the values of the input variables, no substitutions on the input variables may occur in the path from $N_{b}$ to $N_{e}$. Because this path should be a loop, $L_{b}^{1}$ must be an ancestor of $L_{e}^{1}$. Finally, a special more general relation for moded atoms must hold between $L_{b}^{1}$ and $L_{e}^{1}$. We will show that these three conditions imply non-termination.

A moded atom $A$ is moded more general than a moded atom $B$, if any atom in the denotation of $A$ is more general than some atom in the denotation of $B$.

Definition 7. A moded atom $A$ is moded more general than a moded atom $B$ w.r.t. a program $P, A \triangleright B$, iff: 
$\forall I \in \operatorname{Den}(A), \exists I^{\prime} \in \operatorname{Den}(B): I$ is more general than $I^{\prime}$

We illustrate this moded more general relation with some small examples.

Example 3. The following relations hold w.r.t. the binary tree program from Example 2.

$-\operatorname{bin}(X) \triangleright \operatorname{bin}(\underline{I})$

The denotation of $\operatorname{bin}(X)$ only contains the atom itself, which is more general than any atom in the denotation of $\operatorname{bin}(\underline{I})$, e.g. $\operatorname{bin}($ empty).

- $\operatorname{bin}(\operatorname{tree}(\operatorname{tree}(\underline{\operatorname{In}}, X n), Y)) \triangleright \operatorname{bin}(\operatorname{tree}(\underline{I}, \operatorname{tree}(X$, empty $)))$

For example, if $\underline{I n}=$ empty, then $\underline{I}=$ tree (empty, empty) yields an atom in the denotation that satisfies the more general relation.

Because the denotation of a moded atom is in general infinite, we cannot check this property for every atom in the denotation. However, there is a syntactic sufficient condition to check if the moded more general relation holds between two given moded atoms $A$ and $B$. The condition is based on a particular kind of unifiability of the atoms.

We introduce the following notations. Let $\operatorname{InVar}_{P}$ be the set of input variables and $\operatorname{Var}_{P}$ the set of normal variables. To every $\underline{I} \in \operatorname{In} \operatorname{Var}_{P}$ we associate a fresh normal variable $I$. Let Term $_{P}^{+}$denote the set of all terms constructible in the underlying language of $P$ augmented with the variables $\left\{I \mid \underline{I} \in \operatorname{InVar}_{P}\right\}$.

Proposition 1. Let $A$ and $B$ be two moded atoms. Let $A_{1}$ and $B_{1}$ be renamings of these atoms such that they have no shared variables. Let $A_{2}$ and $B_{2}$ denote variants of $A_{1}$ and $B_{1}$ in which every input variable $\underline{I}$ is replaced by $I$. Let $N_{1}^{a}, \ldots, N_{n}^{a}$ be a subset of the normal variables in $A_{1}$ and $I_{1}^{b}, \ldots, I_{m}^{b}$ be the fresh variables associated to the input variables in $B_{2}$.

If $A_{2}$ and $B_{2}$ are unifiable with a substitution $\gamma=\left\{N_{1}^{a} \backslash t_{1}, \ldots, N_{n}^{a} \backslash t_{n}\right.$, $\left.I_{1}^{b} \backslash t_{1}^{+}, \ldots, I_{m}^{b} \backslash t_{m}^{+}\right\}$with $t_{1}, \ldots, t_{n} \in$ Term $_{P}$ and $t_{1}^{+}, \ldots, t_{m}^{+} \in$ Term $_{P}^{+}$, then $A$ is moded more general then $B$.

Proof. Let $\alpha=\left\{N_{1}^{a} \backslash t_{1}, \ldots, N_{n}^{a} \backslash t_{n}\right\}$ and $\beta=\left\{I_{1}^{b} \backslash t_{1}^{+}, \ldots, I_{m}^{b} \backslash t_{m}^{+}\right\}$. Because $I_{1}^{b}, \ldots, I_{m}^{b}$ can not occur in $t_{1}, \ldots, t_{n}, \gamma=\beta \circ \alpha$, and by unifiability, $A_{2} \alpha \beta=$ $B_{2} \alpha \beta$. Moreover, since $B_{2}$ does not contain $N_{1}^{a}, \ldots, N_{n}^{a}, B_{2} \alpha \beta=B_{2} \beta$, and since $A_{2} \alpha$ does not contain $I_{1}^{b}, \ldots, I_{m}^{b}, A_{2} \alpha \beta=A_{2} \alpha$. Thus, $A_{2} \alpha=B_{2} \beta$.

Let $A_{c}$ be an element of $\operatorname{Den}\left(A_{1}\right)$. Then, there exists a substitution $\psi=$ $\left\{I_{1}^{a} \backslash s_{1}, \ldots, I_{k}^{a} \backslash s_{k}\right\}$, where $\underline{I_{1}^{a}}, \ldots, \underline{I_{k}^{a}}$ are all input variables of $A_{1}, s_{1}, \ldots, s_{k} \in$ Term $_{P}$ and $s_{1}, \ldots, s_{k}$ are ground, such that $A_{c}=A_{2} \psi$.

Now consider the atom $B_{c}=B_{2} \beta \psi$. First, $B_{c} \in \operatorname{Den}\left(B_{1}\right)$. This is because $\beta$ replaces all $I_{j}^{b}$ of $B_{2}$ by terms $t_{j}^{+}$. These terms $t_{j}^{+}$may contain variables $I_{l}^{a}$ of $A_{2}$, but these are all substituted to ordinary ground terms $s_{l} \in \operatorname{Term}_{P}$ by $\psi$.

Finally, $A_{c} \alpha=A_{2} \psi \alpha=A_{2} \alpha \psi=B_{2} \beta \psi=B_{c}$. Note that $A_{2} \psi \alpha=A_{2} \alpha \psi$ because no $s_{i}$ of $\psi$ can contain a variable $N_{j}^{a}$ of $\alpha$, nor can any $t_{i}$ of $\alpha$ contain a variable $I_{j}^{a}$ of $\psi$. Thus $A_{c}$ is more general than an element of $\operatorname{Den}\left(B_{1}\right)$.

Example 4. The moded atoms of the last example are already variable disjunct. To check if the moded more general relation holds, we have to check if the atoms are unifiable with a substitution of the correct forms. 
- $\operatorname{bin}(X)=\operatorname{bin}(I)$ with substitution: $\{I \backslash X\}$

- bin $(\operatorname{tree}(\operatorname{tree}(I n, X n), Y))=\operatorname{bin}(\operatorname{tree}(I, \operatorname{tree}(X$, empty $)))$ with substitution: $\{I \backslash \operatorname{tree}(I n, X n), Y \backslash$ tree $(X$, empty $)\}$

\subsection{Non-termination of Moded More General Loops}

If a moded SLDNF-derivation contains a path without substitutions on input variables, such that the ancestor relation and the moded more general relation hold between the first and last selected literal in that path, we call this path a moded more general loop. We will show that a moded more general loop implies non-termination.

Definition 8. In a moded $S L D N F$-derivation $D$, nodes $N_{i}: G_{i}$ and $N_{j}: G_{j}$ are a moded more general loop, $N_{i}: G_{i} \stackrel{m m g}{\rightarrow} N_{j}: G_{j}$, iff:

- No substitutions on input variables occur in the path from $N_{i}$ to $N_{j}$.

- $L_{i}^{1} \prec$ anc $L_{j}^{1}$.

$-L_{j}^{1} \triangleright L_{i}^{1}$

Note that when no confusion can occur, we may omit writing the goal in the moded more general loop.

A moded more general loop, $N_{i}: G_{i} \stackrel{m m g}{\rightarrow} N_{j}: G_{j}$, corresponds to an infinite loop for every concrete goal in the denotation of $G_{i}$.

Theorem 1 (Sufficiency of the moded more general loop). Let $N_{i}$ : $G_{i} \stackrel{m m g}{\rightarrow} N_{j}: G_{j}$ be a moded more general loop in a moded SLDNF-derivation $D$ of a program $P$ and a moded query $I$. The sequence of clauses from $N_{i}$ to $N_{j}$, $\left\langle C_{1}, \ldots, C_{n}\right\rangle$, can be repeated infinitely often for any goal in $\operatorname{Den}\left(G_{i}\right)$.

Proof. Because $L_{i}^{1}$ is an ancestor of $L_{j}^{1}$, only the selected literal of $N_{i}$ influences if the sequence of clauses can be repeated infinitely often.

Because no substitutions on input variables occur in the path from $N_{i}$ down to $N_{j},\left\langle C_{1}, \ldots, C_{n}\right\rangle$ is applicable to any atom in $\operatorname{Den}\left(L_{i}^{1}\right)$. Obviously, this path is also applicable to any atom $A$, which is more general than some atom $B$ in $\operatorname{Den}\left(L_{i}^{1}\right)$. Furthermore, after applying $\left\langle C_{1}, \ldots, C_{n}\right\rangle$ to $A$, the resulting selected literal is more general than the selected literal after applying $\left\langle C_{1}, \ldots, C_{n}\right\rangle$ to $B$.

As $L_{j}^{1} \triangleright L_{i}^{1}$, any atom in $\operatorname{Den}\left(L_{j}^{1}\right)$ is more general than some atom in $\operatorname{Den}\left(L_{i}^{1}\right)$.

Therefore, let $S$ be the union of $\operatorname{Den}\left(L_{i}^{1}\right)$ and all more general atoms. Then, $\left\langle C_{1}, \ldots, C_{n}\right\rangle$ is applicable to any atom of $S$, and after applying these clauses, the selected literal of the resulting goal is again an atom of $S$. Thus, this sequence of clauses is infinitely often applicable to elements of $S$.

We illustrate this non-termination condition with our running example.

Example 5 (Non-termination proof of binary tree). Let us revisit Example 1 with a query $\operatorname{bin}(X)$. The SLDNF-tree constructed by LP-check for this program and query is almost the same as in Figure 1(a). The only difference is that the input variables are replaced by ordinary variables. 
The constructed SLDNF-tree satisfies the conditions of Definition 8 , so $N_{0} \stackrel{m m g}{\longrightarrow}$ $N_{2}$ is a moded more general loop. Therefore, non-termination of this example is proven by Theorem 1 .

Observe that Theorem 1 can straightforwardly be generalized to conclude nontermination for any goal that is more general than an element of $\operatorname{Den}\left(G_{i}\right)$. In particular, the analysis is not restricted to goals with ground inputs: Theorem 1 also holds for an "extended" denotation of $G_{i}$, with non-ground inputs.

\subsection{Input-Generalizations}

Our experimental evaluation (see Section 4) shows that for many non-terminating programs, non-termination can be proven using the moded more general loop. But, the next example shows that there is room for further improvement.

Example 6 (Termination behavior of flat).

flat (niltree, nil).

flat (tree(X, niltree, XS), cons (X, YS)) :- flat (XS, YS).

flat (tree (X, tree (Y, YS1, YS2), XS), ZS) :-

flat (tree (Y, YS1, tree (X, YS2, XS)), ZS).

This program, flat, flattens a binary tree into a list denoted with the cons notation. To flatten the tree, the program repeatedly moves one element from the left to the right subtree until the left subtree is empty. When the left subtree is empty, we proceed by processing the right subtree. If the first argument of the query is a variable, this program loops w.r.t. the third clause.

Figure 2 shows a part of the moded generalized SLDNF-tree constructed for moded query $f l a t(T, \underline{I})$ using LP-check with repetition number 3 . No nodes in the derivations satisfy Definition 8. The reason for this is that we replace a variable by a compound term when applying the third clause.

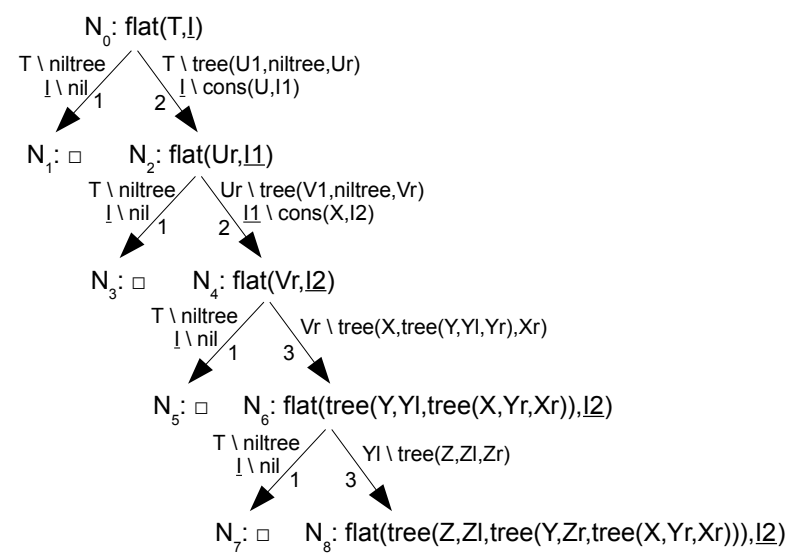

Fig. 2. Moded generalized SLDNF-tree with LP-check of flat (Example 6) 
To prove non-termination for programs such as flat, we define an inputgeneralization. This input-generalization is such that proving non-termination of an input-generalized goal implies non-termination of the original goal.

Definition 9. We say that $A^{\alpha}$ is an input-generalization of an atom $A$, if there exist terms $t_{1}, \ldots, t_{n}$ in $A$ and fresh input variables $I_{1}, \ldots, I_{n}$ such that $A^{\alpha}=$ $A\left(\underline{I_{1}} \rightarrow t_{1}, \ldots, \underline{I_{n}} \rightarrow t_{n}\right)$ and $\operatorname{Var}\left(A^{\alpha}\right) \cap \operatorname{Var}\left(\left(t_{1}, \ldots, \overline{t_{n}}\right)\right)=\bar{\emptyset}$.

Example 7 (Input generalizations).

- $\operatorname{bin}\left(\operatorname{tree}\left(\underline{I}, \underline{I}_{1}\right)\right)$ is an input-generalization of $\operatorname{bin}(\operatorname{tree}(\underline{I}, \operatorname{tree}(X, \operatorname{empty})))$

- $\operatorname{bin}\left(\underline{I_{2}}\right)$ is an input-generalization of $\operatorname{bin}\left(\operatorname{tree}\left(\underline{I}, \underline{I_{1}}\right)\right)$

- $\operatorname{bin}\left(\operatorname{tr} e e\left(\underline{I_{3}}, X\right)\right)$ is not an input generalization of $\operatorname{bin}(\operatorname{tree}(\operatorname{tree}(X, Y), X))$ This last example refers to the condition of the empty intersection of the variable sets. We return to this condition in Example 8.

To check if a path is non-terminating w.r.t. an input-generalized goal, we define an input-generalized derivation. This derivation is constructed by applying a path in a given derivation to the input-generalized selected literal of the first node in the path.

Definition 10. Let $D$ be a moded SLDNF-derivation $N_{i}, \ldots, N_{j}$, such that $L_{i}^{1} \prec$ anc $L_{j}^{1}$. Let $\left\langle C_{1}, \ldots, C_{n}\right\rangle$ be the sequence of clauses applied from $N_{i}$ to $N_{j}$ and let $A^{\alpha}$ be an input-generalization of $L_{i}^{1}$.

The input-generalized derivation $D^{\prime}$ for $A^{\alpha}$, is constructed by applying the sequence of clauses $\left\langle C_{1}, \ldots, C_{n}\right\rangle$ to $A^{\alpha}$. The input-generalized nodes $N_{i}^{\alpha}$ and $N_{j}^{\alpha}$ are the top and bottom nodes of $D^{\prime}$, respectively.

Next, we prove that non-termination of the input-generalized derivation implies non-termination of the original goal. First we introduce two lemmas.

Lemma 1. Let $A^{\alpha}$ be an input generalization of $A$, then $A \triangleright A^{\alpha}$.

Proof. Let $\underline{I_{1}}, \ldots, \underline{I_{n}}$ be the input variables of $A$ and $I_{n+1}, \ldots, \underline{I_{m}}$ be the new introduced input variables in $A^{\alpha}$. For every concrete atom $\bar{A}_{c}$ in $\overline{\operatorname{Den}}(A), \underline{I_{1}}, \ldots, \underline{I_{n}}$ are replaced by ground terms. To construct an atom $A_{c}^{\alpha}$ of $\operatorname{Den}\left(A^{\alpha}\right)$, for which $A_{c}$ is more general then $A_{c}^{\alpha}$, one replaces $\underline{I}_{1}, \ldots, \underline{I_{n}}$ by the same values as in $A_{c}$ and $\underline{I}_{n+1}, \ldots, \underline{I_{m}}$ by instances of the corresponding terms, $t_{n+1}, \ldots, t_{m}$, in $A_{c}$. Due to the condition that $\operatorname{Var}\left(A^{\alpha}\right) \cap \operatorname{Var}\left(\left(t_{n+1}, \ldots, t_{m}\right)\right)=\emptyset, A_{c}$ is more general than $A_{c}^{\alpha}$.

Example 8. To explain the condition on the intersection of the variables in Definition 9, consider the atom $A=a(X, f(X))$. If we omit the condition on the variables, we could consider $A^{\alpha}=a(X, \underline{I})$ as an input generalization. $\operatorname{Den}(A)=\{a(X, f(X))\}$ and $a(X, f(X))$ is not more general than any element in $\operatorname{Den}(a(X, \underline{I}))$. So, the property that $A \triangleright A^{\alpha}$ would not hold.

Lemma 2. Let $A$ and $B$ be atoms such that $A \triangleright B$ and let every atom in $\operatorname{Den}(B)$ be non-terminating w.r.t. a program $P$, then, every atom in $\operatorname{Den}(A)$ is nonterminating w.r.t. $P$. 
Proof. Every atom of $\operatorname{Den}(A)$ is more general than a non-terminating atom.

Corollary 1 (Non-termination with input-generalization). Let $N_{i}: G_{i}$ and $N_{j}: G_{j}$ be nodes in a derivation $D$ of a program $P$ for a moded query $I$, such that $L_{i}^{1} \prec_{\text {anc }} L_{j}^{1}$, and let $N_{i}^{\alpha}$ and $N_{j}^{\alpha}$ be input-generalized nodes in an input-generalized derivation $D^{\prime}$ of $N_{i}$ and $N_{j}$ for $A$.

If $N_{i}^{\alpha} \stackrel{m m g}{\rightarrow} N_{j}^{\alpha}$, then every concrete goal in the denotation of $G_{i}$ is nonterminating w.r.t. program $P$.

Proof. Follows from Theorem [1 and the two previous lemmas.

We illustrate these input-generalizations by revisiting the flat example.

Example 9 (Non-termination of flat). To prove non-termination, we generalize node $N_{6}$ to flat $(\operatorname{tree}(Y, Y l, \underline{I n}), \underline{I 2})$, by changing the subterm tree $(X, Y r, X r)$ to a new input variable $\underline{I n}$.

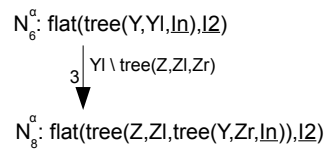

Fig. 3. Input-generalized SLDNF-derivation of flat

Figure 3, shows the input-generalized moded SLDNF-derivation for flat $(\operatorname{tree}(Y, Y l, \underline{I n}), \underline{I 2})$. This derivation is a moded more general loop: $N_{6}^{\alpha} \stackrel{m m g}{\rightarrow}$ $N_{8}^{\alpha}$. Therefore, non-termination of the program flat w.r.t. the concrete goals in the denotation of the goal of $N_{6}$ is proven by Corollary 1 .

Note that a concrete query in the denotation of a moded query might not reach the moded more general loop. However, classes of non-terminating top level queries can be obtained by applying all substitutions on the input variables between the root and the first node of the moded more general loop. In the last example, this class of top level queries is $\operatorname{flat}(T, \operatorname{cons}(\underline{U}, \operatorname{cons}(\underline{X}, \underline{I 2})))$.

\section{Experimental Evaluation}

To evaluate our approach, we implemented a non-termination analyzer $P 2 P$, from Prediction to Proof, based on Corollary 1. We tested $P 2 P$ on a benchmark of 48 non-terminating pure logic programs. First, we describe our analyzer and the benchmark. Then, we compare our tool with the non-termination inference tool NTI [10].

\subsection{P2P: From Prediction to Proof}

We implemented $P 2 P$ in SWI-prolog. $P 2 P$ is freely available 3 and consists of two components. First, the implementation of the termination prediction

\footnotetext{
${ }^{2}$ Homepage of SWI-prolog: http://www.swi-prolog.org/

${ }^{3}$ Available at http://www.cs.kuleuven.be/ $\operatorname{dean} / \mathrm{p} 2 \mathrm{p} . \mathrm{html}$
} 
approach [12, TPoLP4, constructs the moded SLDNF-derivation and predicts the termination behavior. If $T$ PoLP predicts a derivation to be non-terminating, the second component tries to prove non-termination in the derivation.

To prove non-termination, $P 2 P$ checks if the derivation contains a moded more general loop or it uses a backtracking search to attempt to construct an input generalized derivation that contains a moded more general loop. Although many input generalizations can be constructed, proving non-termination in a derivation can be done rather efficiently. This is because the LP-cuts made by $T P o L P$ correctly identify an infinite loop if the repetition number is sufficiently high. Therefore, instead of checking the conditions of the moded more general loop between all pairs of nodes in the derivation, it suffices to check these conditions for the pairs of nodes of the LP-cut.

\subsection{Benchmark of Termination Problems}

Our benchmark consists of the non-terminating pure logic programs from the termination competition of 2007. The benchmark and the results from the tools that participated in the competition are available5. The benchmark of the termination competition contains around 300 logic programs and moded queries representing different challenges in termination and non-termination analysis. A few programs from the competition are omitted because they contain non-logical operations such as arithmetics. The competition benchmark contains some doubles. These were also omitted. The benchmark contains 48 non-terminating programs. All programs contain between 2 and 15 clauses, except for binary4, which contains 41 clauses. The only other non-termination analyzer, NTI [10], proves non-termination for 45 benchmark programs.

Table 1 shows our experimental evaluation on this benchmark using LP-check with pruning, with 4 as a repetition number. The result of our tool is given in the column $P 2 P, V$ denotes that non-termination is proven while $X$ denotes that no non-termination proof was found. The result of $N T I$ is given in the column NTI. The columns Size and Time show the size in the number of nodes of the SLDNF-tree and the analysis time in seconds, respectively.

The results are very satisfactory. For all programs in the benchmark, nontermination is proven and a class of non-terminating queries can be constructed. The analyzer is very fast. Any benchmark program is analyzed in less than a second and the memory use never exceeds a few megabytes.

As stated, these experiments have been performed using 4 as a repetition number. When we use 3 as a repetition number, our tool fails to prove nontermination of programs $p l 7.6 .2 . a$ and $p l 7.6 .2 . b$. These are two erroneous implementations of a path find algorithm. When using 2 as repetition number, proving non-termination fails for about $25 \%$ of the benchmark programs.

\footnotetext{
${ }^{4}$ Available at http://www.cs.kuleuven.be/ dean/termination_prediction.html

${ }^{5}$ Available at http://www.lri.fr/ ${ }^{\text {marche/termination-competition/ }}$
} 
Table 1. Benchmark of non-terminating pure logic programs

\begin{tabular}{|l|c|c|c|c||l|l|l|l|l|}
\hline Name program & $P 2 P$ & Size & Time & $N T I$ & Name program & $P 2 P$ & Size & Time & $N T I$ \\
\hline ackermann-ioi & $\mathrm{V}$ & 9 & 0.33 & $\mathrm{~V}$ & permutation-fb & $\mathrm{V}$ & 22 & 0.26 & $\mathrm{~V}$ \\
bad sublist & $\mathrm{V}$ & 33 & 0.29 & $\mathrm{~V}$ & pl1.1 & $\mathrm{V}$ & 8 & 0.25 & $\mathrm{~V}$ \\
binary4 & $\mathrm{V}$ & 12 & 0.27 & $\mathrm{~V}$ & pl3.1.1 & $\mathrm{V}$ & 12 & 0.30 & $\mathrm{~V}$ \\
delete-bff & $\mathrm{V}$ & 13 & 0.31 & $\mathrm{~V}$ & pl3.5.6 & $\mathrm{V}$ & 13 & 0.31 & $\mathrm{~V}$ \\
der-fb & $\mathrm{V}$ & 22 & 0.29 & $\mathrm{~V}$ & pl4.0.1-oooi & $\mathrm{V}$ & 33 & 0.27 & $\mathrm{~V}$ \\
doublehalfpred & $\mathrm{V}$ & 38 & 0.28 & $\mathrm{~V}$ & pl4.5.2 & $\mathrm{V}$ & 481 & 0.36 & $\mathrm{~V}$ \\
example4-2 & $\mathrm{V}$ & 4 & 0.23 & $\mathrm{~V}$ & pl4.5.3a & $\mathrm{V}$ & 10 & 0.29 & $\mathrm{~V}$ \\
flatlength-fbf & $\mathrm{V}$ & 14 & 0.23 & $\mathrm{~V}$ & pl4.5.3b & $\mathrm{V}$ & 10 & 0.24 & $\mathrm{~V}$ \\
flatlength-ffb & $\mathrm{V}$ & 19 & 0.23 & $\mathrm{~V}$ & pl4.5.3c & $\mathrm{V}$ & 11 & 0.27 & $\mathrm{~V}$ \\
flat-oi & $\mathrm{V}$ & 9 & 0.26 & $\mathrm{X}$ & pl5.2.2 & $\mathrm{V}$ & 59 & 0.27 & $\mathrm{~V}$ \\
frontier-fb & $\mathrm{V}$ & 12 & 0.27 & $\mathrm{~V}$ & pl7.6.2.a & $\mathrm{V}$ & 39 & 0.27 & $\mathrm{X}$ \\
ifdiv & $\mathrm{V}$ & 19 & 0.29 & $\mathrm{~V}$ & pl7.6.2.b & $\mathrm{V}$ & 45 & 0.33 & $\mathrm{X}$ \\
in-bf & $\mathrm{V}$ & 18 & 0.29 & $\mathrm{~V}$ & quicksort-fb & $\mathrm{V}$ & 72 & 0.26 & $\mathrm{~V}$ \\
inorder-fb & $\mathrm{V}$ & 4 & 0.27 & $\mathrm{~V}$ & quicksort-oi & $\mathrm{V}$ & 74 & 0.26 & $\mathrm{~V}$ \\
insert-bff & $\mathrm{V}$ & 22 & 0.29 & $\mathrm{~V}$ & reverse-fb & $\mathrm{V}$ & 9 & 0.32 & $\mathrm{~V}$ \\
log2a-oi & $\mathrm{V}$ & 35 & 0.25 & $\mathrm{~V}$ & select-bff & $\mathrm{V}$ & 8 & 0.32 & $\mathrm{~V}$ \\
log2b-oi & $\mathrm{V}$ & 29 & 0.28 & $\mathrm{~V}$ & slowsort-fb & $\mathrm{V}$ & 123 & 0.27 & $\mathrm{~V}$ \\
mapcolor & $\mathrm{V}$ & 23 & 0.31 & $\mathrm{~V}$ & slowsort-oi & $\mathrm{V}$ & 26 & 0.26 & $\mathrm{~V}$ \\
member-bf & $\mathrm{V}$ & 8 & 0.27 & $\mathrm{~V}$ & sublist-bf & $\mathrm{V}$ & 30 & 0.21 & $\mathrm{~V}$ \\
mergesort & $\mathrm{V}$ & 171 & 0.28 & $\mathrm{~V}$ & subset-bf & $\mathrm{V}$ & 21 & 0.23 & $\mathrm{~V}$ \\
mergesort-oi & $\mathrm{V}$ & 54 & 0.28 & $\mathrm{~V}$ & subset-fb & $\mathrm{V}$ & 14 & 0.26 & $\mathrm{~V}$ \\
mergesort_variant & $\mathrm{V}$ & 15 & 0.23 & $\mathrm{~V}$ & suffix-bf & $\mathrm{V}$ & 9 & 0.25 & $\mathrm{~V}$ \\
minimum-fb & $\mathrm{V}$ & 8 & 0.29 & $\mathrm{~V}$ & transpose2 & $\mathrm{V}$ & 6 & 0.28 & $\mathrm{~V}$ \\
naive reverse-fb & $\mathrm{V}$ & 8 & 0.37 & $\mathrm{~V}$ & tree_member-bf & $\mathrm{V}$ & 12 & 0.28 & $\mathrm{~V}$ \\
\hline
\end{tabular}

\subsection{Comparison with NTI}

To infer non-terminating queries, NTI first transforms a given program into a binary program using binary unfoldings. Then, it compares the head and body of the clauses in the binary program with a special more general relation. If this relation holds, non-termination is proven.

The binary unfolding of a program represent the calls made during program execution. Thus, it corresponds to comparing the selected literals in our symbolic computation. The binary unfolding of a program can be computed using a fixpoint operator.

The special more general relation used by NTI, $\triangle$-more general, is based on the notion of derivation neutral (DN) filters. These filters are functions defining, for a clause and argument position, which terms have no influence on the applicability of the clause. Furthermore, if the head atom satisfies the filter, the body atom must satisfy the filter as well. We explain NTI's non-termination condition and compare it with our approach using some small examples.

\section{Example 10 (Recursive clause of reverse-fb). $\operatorname{rev}([\mathrm{H} \mid \mathrm{T}], \mathrm{Temp}, \operatorname{Res}):-\operatorname{rev}(\mathrm{T},[\mathrm{H} \mid \mathrm{Temp}]$, Res $)$.}

In this clause, the second argument is not replaced by a more general one. Therefore, NTI needs a DN filter to prove non-termination. The applicability of the clause does not depend on the value of Temp, so we can use the trivial filter, instance of $X$, for the second argument position. We can also use this filter for the last argument position. Therefore, NTI concludes that this clause is nonterminating for each goal where the first argument is more general than $[H \mid T]$ and the second and third argument are instances of $X$. 
These DN filters cannot depend on the names of the variables. Therefore, they cannot express that two argument must contain a common subterm.

Example 11 (Variable independent filters).

$\mathrm{a}(\mathrm{X}, \mathrm{X}):-\mathrm{a}(\mathrm{s}(\mathrm{X}), \mathrm{s}(\mathrm{X}))$.

Both arguments are replaced by more specific ones and the applicability does not depend on the value of $X$. However, since both arguments must be bound to the same term, NTI fails to prove non-termination of this example.

Instead of comparing all the arguments independently, our approach compares the selected literals. Therefore, our condition does not have this restriction.

Because NTI requires that each argument is either replaced by a more general one or satisfies a DN filter, NTI fails to prove non-termination if in one argument, a subterm is replaced by a more general one while another subterm is replaced by a more specific one. This is because of the requirement that if the head atom satisfies the filter, the body atom needs to satisfy it as well.

Example 12 (Looping clause of flat-oi). In the third clause of flat-oi in Example 9. the first argument of this clause contains two such subterms. XS is replaced by tree (X, YS2, XS) and tree(Y, YS1, YS2) is replaced by YS1.

Because we allow arguments to contain both input and ordinary variables, our condition does not have this restriction.

Table 1 shows that NTI fails to prove non-termination of 3 programs. These 3 programs are examples of the two classes of problems that are illustrated by Examples 11 and 12. The actual results on the termination competition were worse for NTI, as we have rewritten some programs that NTI could not parse.

\section{Conclusion and Future Work}

We introduced a new approach to non-termination analysis of logic programs based on a finite, symbolic derivation tree for a moded query. This symbolic tree represents the derivation trees of all concrete queries denoted by the moded query. To prove non-termination we look for a loop in this symbolic derivation tree. We implemented this approach and evaluated it on a benchmark of 48 non-terminating programs from the termination competition of 2007. Our tool, $P 2 P$, proves non-termination of all benchmark programs. We have shown that our technique improves on the results of the only other non-termination analyzer, $N T I$, and that we can handle 2 new classes of programs.

A class of programs that we currently cannot handle, are programs that require types to describe the looping goals. These are programs of the following form:

$\mathrm{p}(\mathrm{L}):-\operatorname{list}(\mathrm{L}), \mathrm{p}([\mathrm{a} \mid \mathrm{L}])$. list ([]).

list $([\mathrm{H} \mid \mathrm{T}]):-\operatorname{list}(\mathrm{T})$. 
We cannot prove that a query $p(\underline{L})$ with $\underline{L}$ an input list is non-terminating, because substitutions on the input variables occur between all selected literals that satisfy the moded more general relation. To overcome this problem, we plan to extend our technique with type analysis [3] and non-failure analysis [5]. For this program and query, the type analysis would infer that all arguments are lists and the non-failure analysis would infer that $\operatorname{list}(L)$ cannot fail if $L$ is a list. If we combine this information, it is clear that the first clause is a loop.

We also plan to extend our technique for programs containing arithmetic expressions by using a finite domain solver to infer domains and initial values such that the arithmetic conditions in the loop will always succeed.

Acknowledgments. We thank the reviewers for their feedback and helpful comments.

\section{References}

1. Bol, R.N.: Loop checking in logic programming. CWI (Centre for Mathematics and Computer Science), Amsterdam, The Netherlands (1995)

2. Bruynooghe, M., Codish, M., Gallagher, J., Genaim, S., Vanhoof, W.: Termination analysis through combination of type based norms. TOPLAS 29(2), 10 (2007)

3. Bruynooghe, M., Janssens, G.: An instance of abstract interpretation integrating type and mode inferencing. In: ICLP/SLP, pp. 669-683 (1988)

4. Codish, M.: Proving termination with (boolean) satisfaction. In: King, A. (ed.) LOPSTR 2007. LNCS, vol. 4915, pp. 1-7. Springer, Heidelberg (2008)

5. Debray, S.K., López-García, P., Hermenegildo, M.V.: Non-failure analysis for logic programs. In: ICLP, pp. 48-62 (1997)

6. Decorte, S., De Schreye, D., Vandecasteele, H.: Constraint-based termination analysis of logic programs. TOPLAS 21(6), 1137-1195 (1999)

7. Lloyd, J.W.: Foundations of Logic Programming, 2nd edn. Springer, Heidelberg (1987)

8. Mesnard, F., Bagnara, R.: Cti: A constraint-based termination inference tool for iso-prolog. TPLP 5(1-2), 243-257 (2005)

9. Nguyen, M.T., De Schreye, D.: Polytool: Proving termination automatically based on polynomial interpretations. In: Puebla, G. (ed.) LOPSTR 2006. LNCS, vol. 4407, pp. 210-218. Springer, Heidelberg (2007)

10. Payet, É., Mesnard, F.: Nontermination inference of logic programs. ACM Transactions on Programming Languages and Systems 28(2), 256-289 (2006)

11. Schneider-Kamp, P., Giesl, J., Serebrenik, A., Thiemann, R.: Automated termination analysis for logic programs by term rewriting. In: Puebla, G. (ed.) LOPSTR 2006. LNCS, vol. 4407, pp. 177-193. Springer, Heidelberg (2007)

12. Shen, Y.-D., De Schreye, D., Voets, D.: Termination prediction for general logic programs. Report CW 2009 536, K.U.L. Accepted for TPLP 